\title{
Spectrogram-based assessment of small SNR variations, with application to medical electrodes
}

\author{
Zeljka Milanović ${ }^{\text {, Nicoletta Saulig }}{ }^{1}$, Ivan Marasović ${ }^{*}$ and Damir Seršić ${ }^{3}$
}

\begin{abstract}
In this paper, the problem of detection of small signal-to-noise ratio (SNR) variations in noisy signals is addressed in order to provide an efficient and fast method for detection of faulty electroencephalogram (EEG) electrodes which can improve the interpretation of medical data. The method for slight SNR variation assessment, based on the estimation of the longest useful information cluster, is proposed as an alternative to commonly used estimators such as signal energy spectral density, spectral peaks, and spectrogram entropy, which exhibited limited reliability for the considered task. The method proposed in this paper is validated on real signals, which are resistance fluctuations of the EEG Corkscrew electrode solder connection, in which failure is typically manifested as a lower signal-to-noise ratio in the output signal, when compared to the valid electrode. In order to obtain a reliable criterion for the distinction of signals with slight SNR variations, a time-frequency method that relies on observation of the longest useful information cluster of data preserved after the K-means-based denoising application has been introduced. Based on the measurement of the longest existing stationary component, an expert system has been developed, which provides reliable failure detection method with detection accuracy of up to $97.6 \%$. Results on real and simulated data show that the proposed method can be adopted as a computer-aided decision system in a wide range of applications requiring high sensitivity to slight variations of SNRs.
\end{abstract}

Keywords: EEG, Failure detection, Entropy, Corkscrew electrodes failure, Time-frequency distributions, K-means

\section{Introduction}

A widely accepted method to determine the reliability and lifetime of a wide range of electronic devices is through testing of the chemical and mechanical properties of the used materials. The goal of such tests is to reliably determine the set on of the failure. In many cases, failure of electronic devices can be caused by degradation (electromigration or oxidization) of metallic interconnects and solder joints which lose their conduction properties. Some known failure detection methods are based on the research of DC resistance fluctuations, material fatigue, corrosion, and mechanical stress [1-3]. Researching solely resistance fluctuations of the material has proven to be unreliable for determining failure appearance as shown in literature that researched resistance fluctuations using

\footnotetext{
${ }^{*}$ Correspondence: ivan.marasovic@fesb.hr

${ }^{2}$ University of Split Faculty of Electrical Engineering, Mechanical Engineering

and Naval Architecture, University of Split, Split, Croatia

Full list of author information is available at the end of the article
}

disordered networks such as large scale random resistor network (RRN) which exploits the fact that faulty electrode presents non-Gaussian behavior and valid does not, which has not been successfully applied as a reliable discrimination criteria [3]. Therefore, in this paper, time-frequency (TF) analysis has been applied to measured and simulated, reference and faulty, EEG electrode tip resistance fluctuation signals. Resistance fluctuations often appear due to the electromigration process and differences in the material properties and its microstructure $[4,5]$. Material defects or chemical impurities that can be inadvertently introduced during manufacturing process, also significantly contribute to materials' resistance fluctuations. The diffusion of solder metal atoms may decrease the cross-section area of the solder contact which increases the local current density and local resistance. The oxide layer that envelops the stainless steel tip acts as a dielectric that has a significant influence on the solder joint overall conductivity. Cold solder between the 
stainless steel tip and the lead wire can appear during the manufacturing procedure of Corkscrew electrodes and cannot be detected immediately but becomes apparent after some time of use. The cold solder often significantly affects the impedance of the electrode and the collected signals' quality. Electrode impedance change results in increased electrode voltage drop, which leads to a larger voltage difference at the amplifier input. Motivated by the previous arguments, this paper investigates the possibility of detection of failure of EEG Corkscrew electrodes, after manufacturing. The aim of this study is to perform detailed computer simulations and develop an expert system for timely failure detection. Simulations are performed for a range of different noise realizations in order to study the influence of noise type and level on spectrograms' entropy. By the spectral analysis of the measured signals, a superposition of additive white Gaussian noise (AWGN) and flicker noise (FN) has been observed [6]. By focusing on steady-state behavior of total resistance, properties of measured resistance fluctuations are analyzed employing the analysis of the information content of the signal in the TF domain.

The simulation results conform to experimental observations, but also provide an important insight into the failure of solder joints. The results obtained by the proposed computational algorithm based on resistance fluctuations TF analysis are discussed as a potential diagnostic tool for medical electrodes quality testing. Section 2, "Methods", describes the measurement setup that has been built for the purpose of measuring resistance fluctuations, including all the methods used to collect the data for the analysis. The following section "Comparison of criteria for detection of faulty electrodes" compares different criteria used when approaching the problem of small SNR and determining the appropriate procedure for the successful failure detection. Section 4 describes the developed model for failure detection based on investigating resistance fluctuations. Section 5 reports results and discussion of the proposed method, with explanation of the rate of success of the detection. Section 6 concludes the paper with explanation of the obtained error rate and future work.

\section{Methods}

In order to determine electrode behavior and electrical characteristics, it is necessary to understand the microstructure of the solder joint created during the manufacturing process. The Corkscrew electrode consists of a needle $(0.6 \mathrm{~mm}$ in diameter $)$ and a lead wire $(1 \mathrm{~m}$ long). In the manufacturing process of Corkscrew electrode, eutectic alloy of $60 \%$ tin and $40 \%$ led (Sn60Pb40) is used as a bonding material (solder) according to DIN1707. The electrodes tip (needle) is made of stainless steel AISI 304 which has the following composition [7]: $14.7 \% \mathrm{Cr}$, $8.3 \% \mathrm{Ni}, 0.053 \% \mathrm{C}, 0.04 \% \mathrm{~N}, 0.48 \% \mathrm{Si}, 1.42 \% \mathrm{Mn}, 0.39 \% \mathrm{Mo}$, and $0.14 \% \mathrm{Cu}$. This type of stainless steel has a thin oxide film that circumfuses the needles' surface. In order to define the structure and impose a model of solder joint in Corkscrew electrode, it is necessary to address the structure of the observed oxide film. It is composed of an outer iron oxide layer and inner mixed iron-chromium oxide layer [7]. As the manufacturing process of EEG electrodes includes soft soldering procedure which uses temperatures in the range of 300 to $350^{\circ} \mathrm{C}$, it is reasonable to assume that thermally grown oxide layer dominates at the tip surface over the passive oxide layer. The measurement setup consists of source-measure unit (SMU) Keithley 2612 which is configured as constant voltage source. In this configuration, as shown in Fig. 1, the "SourceMeter" instrument functions as a low-impedance voltage source with current limit capability which can measure current

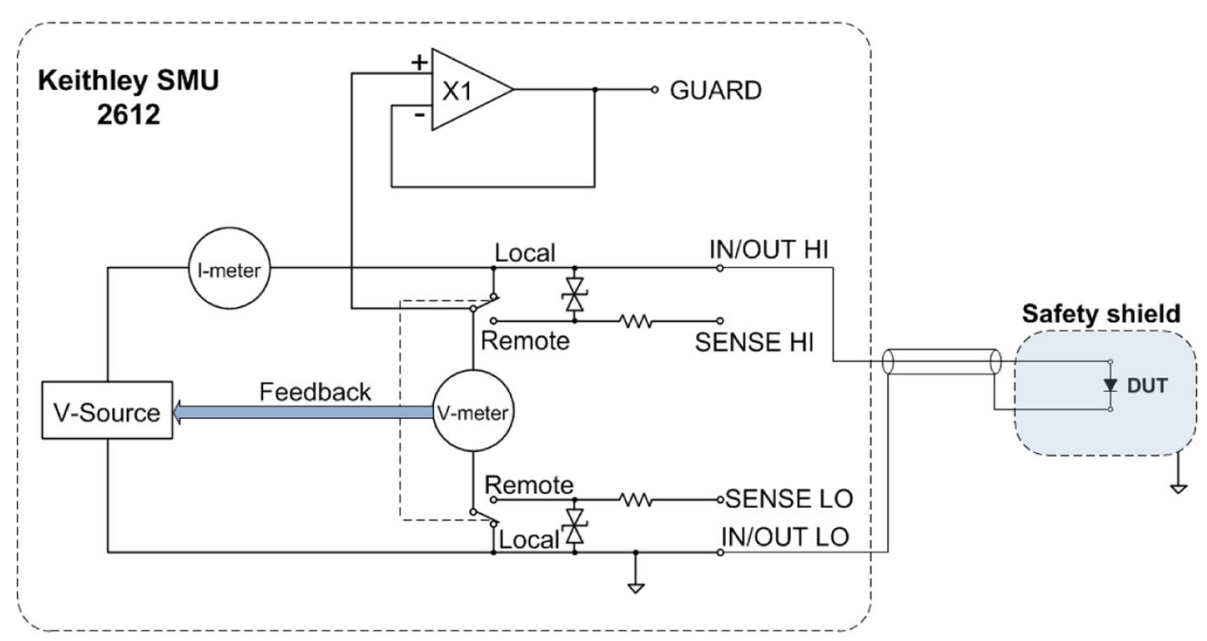

Fig. 1 Measurement setup: Keithley source-measure unit configured as a constant voltage source with a safety shield [3] 
(I-Meter) or voltage (V-Meter). The V-Meter senses the voltage at the input/output terminals (2-wire local sense) and compares it to the pre-programmed voltage level. Additional circuitry is used for continuous monitoring of the output voltage and adjustment to the V-Source if needed. To eliminate the external noise, coaxial cable and a safety shield are used. In this measurement setup, the device under testing (DUT-EEG electrode) is connected as a floating load to reduce the shield current and existent magnetic field. All measurements consist of $P=$ 5000 samples for total recording time of $7 \mathrm{~s}$ and sampling frequency of $714 \mathrm{~Hz}$.

\section{Comparison of criteria for detection of faulty electrodes}

\subsection{Spectral energy, spectral peak, and TF entropy}

The spectra of valid and faulty electrodes are given by the discrete Fourier transform of the signal $x_{n}[p]$, being the resistance fluctuations of one Corkscrew electrode's tip, $n=1: 1: N$, with parameters reported in Section 2 . Figure $2 a$ and $b$ show the energy spectral densities of the resistance fluctuations of the Corkscrew electrodes provided by the measurement setup presented previously. It can be seen that no significant difference between the energy spectral densities of valid and faulty electrodes can be observed. Thus, an alternative approach has been considered, based on the analysis of the electrodes response to an intentionally added low-energy stationary component.

It has been observed that stationary components in the resistance fluctuations can occur as results of nonfiltered network harmonics. Figure $2 \mathrm{c}$ shows the spectral response of one valid, while Fig.2d of one faulty electrode. The addition of a stationary component is manifested as a prominent peak around $250 \mathrm{~Hz}$ in the signal energy spectral density. The addition of a stationary signal component evokes the possibility of considering an approach aimed to detect the presence of a deterministic signal in a noisy environment by means of the signal's energy [8-11]. In cited work the presence or absence of a deterministic signal in a noisy environment assessment is based on the energy of the observed signal: relying on a certain threshold [8-11] on the signal energy, the presence of a deterministic signal is confirmed or denied. Figure 3a shows the energies of signals with stationary component of the valid and faulty electrodes (each set consists of $N=7$ measured electrodes labelled by the manufacturer as either faulty or valid, determined by the appearance of cold solder). It can be noticed that for both sets very similar results are obtained, showing the unsuitability of energy detection for classification purposes for the presented process. Figure $3 \mathrm{~b}$ reports the maximal values, at the stationary component's frequency, of the energy spectral density for valid and faulty electrodes, indicating that valid electrodes tend to better preserve the component peak. However, cases when opposite responses are obtained are not rare, which motivated the search for a more reliable discrimination criterion. Figure 3c shows global entropy values for measured electrodes.

This research suggests that classic spectral analysis is not adequate for the distinction of valid from faulty electrode's signals. An alternative representation of the measured signals is provided by the time-frequency (TF) signal representation. Time-frequency signal representations represent a versatile tool for signal analysis and, in the past few decades, has been applied to a wide range of engineering applications [12-18]. The discrete Quadratic time-frequency distribution (TFD) of an analytic signal $z[p]$ can be written as [19]:

$$
\rho[p, m]=\underset{l \rightarrow m}{\operatorname{DFT}}\left\{G[p, l] \underset{n}{*}\left(z[p+l] z^{*}[p-l]\right)\right\} ; l \in\langle L\rangle
$$

where $G[p, l]$ represents the time-lag kernel. One of the most routinely used time-frequency distributions is the spectrogram, obtained as the squared magnitude of the Fourier transform over a short analyzing window $w(p)$ [19-21]. The spectrogram is thus characterized by the time-lag kernel [19]

$$
G[p, l]=w[p+l] w[p-l] .
$$

Due to its realness, strict positivity, and absence of interferences in the case of non-overlapping components, the spectrogram provides a simple and reliable interpretation of the signal structure in the joint TF domain. Spectrograms of the measured signals, obtained using Hamming window of duration $\mathrm{T} / 10$, where $\mathrm{T}$ is the number of signal samples, are shown in Figs. 4a and 5a. In an attempt to find a suitable criterion for the development of an expert system for assessment of electrodes' validity, the spectrogram complexity has been estimated by the spectrogram's Rényi entropy. The generalized Rényi entropy reads [22]

$$
H_{\alpha}(\rho[p, m]):=\frac{1}{1-\alpha} \log _{2} \sum_{P} \sum_{M}\left(\frac{\rho[p, m]}{\sum_{P} \sum_{M} \rho[p, m]}\right)^{\alpha}
$$

where $\alpha$ is the order of the Rényi entropy (in this paper the value of $\alpha=3$ has been adopted [23, 24]). Since the TF Rényi entropy is an estimator of the TFD complexity, disorder, and information content, intuitively it can be expected that signals with lower SNRs will generally present larger entropy values $[25,26]$, which can be exploited as a discrimination criterion for validity electrodes' assessment [27]. However, the obtained results show tendency of valid electrode to preserve a more concentrated TF structure, and thus smaller entropy values, even if this appears to be quite an unstable indicator, Fig. 3c. 


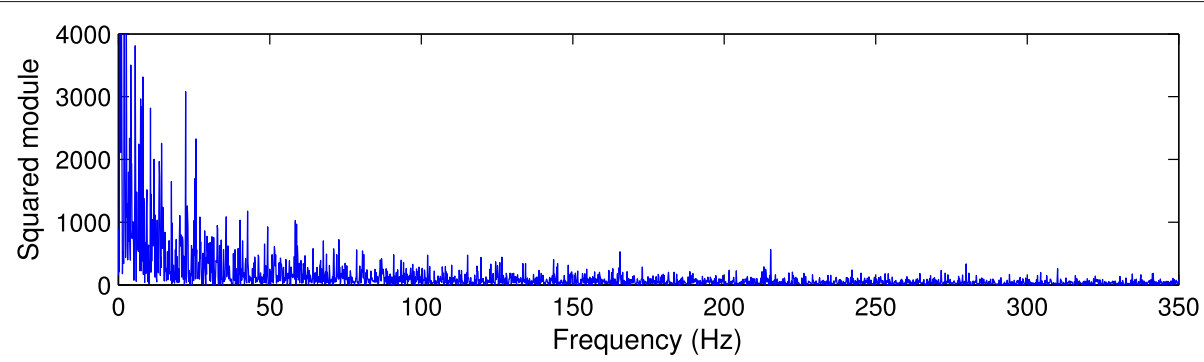

(a)

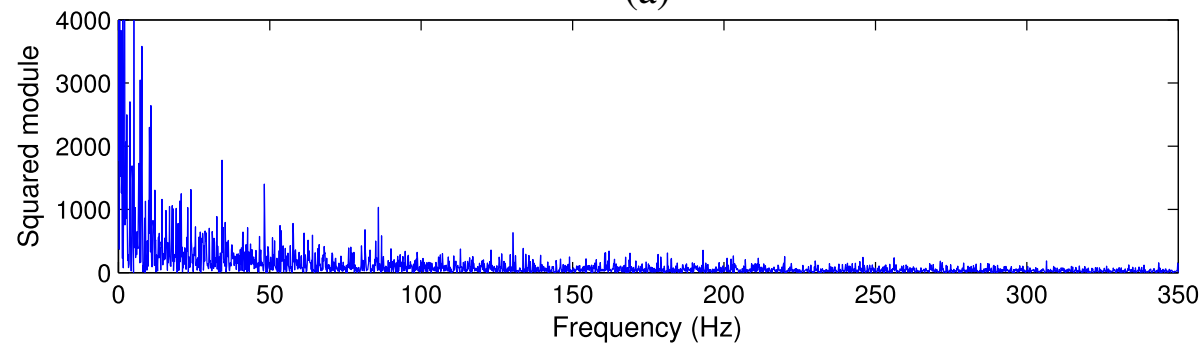

(b)

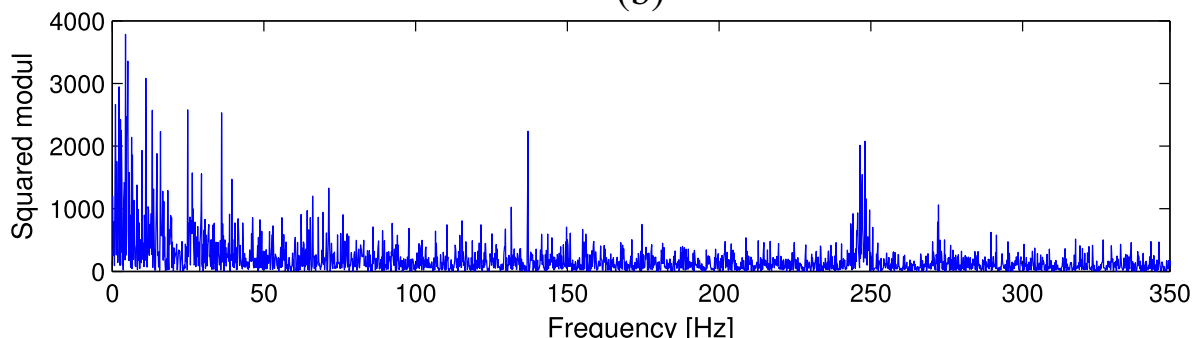

(c)

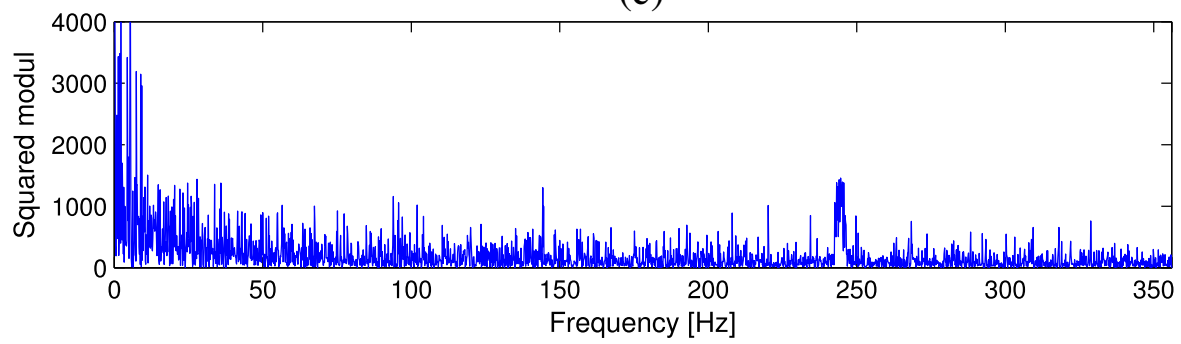

(d)

Fig. 2 Measured signal energy spectral density of $\mathbf{a}$ valid and $\mathbf{b}$ faulty electrode and signal energy spectral density with added stationary component for c valid and $\mathbf{d}$ faulty electrode

\subsection{The criterion based on deterministic signal's continuity}

Since it is known that signal components, as opposed to the noise, are continuous energy regions in the TF plane $[28,29]$, we have proceeded in the search for a robust and reliable method for automatic electrodes' validity assessment by focusing on observation of the components continuity in TF domain, by assuming that lower SNR will cause oscillations in the component amplitude, degrading its continuity. Thus, the proposed method for distinction of faulty from valid electrodes, which can be generally applied to problems of assessing small differences in SNR in as set of measurements, estimates the continuity of the signal component in the TF plane.

The first step of the proposed method consists of denoising the spectrogram $\rho_{n}(p, m)$ of each signal $z_{n}(p)$ using a K-means-based algorithm as described in sequel $[27,30,31]$. The denoising procedure relies on the fact that an amplitude discrimination of data in a TFD can be performed by the K-means algorithm [30]. Considering the set of observations $C_{n}=\left\{\rho_{n}(p, m) \mid p=1, \ldots, P, m=\right.$ $1, \ldots, M\}$, the K-means algorithm partitions these $P \times M$ observations into $K$ subsets

$$
C_{n}=\left\{C_{n, k} \mid k \in \mathbb{N}, 1 \leq k \leq K\right\}
$$




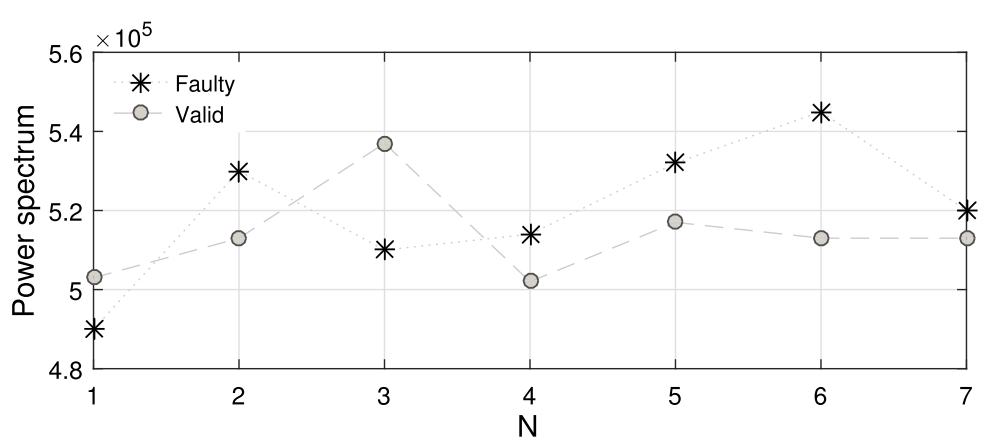

(a)

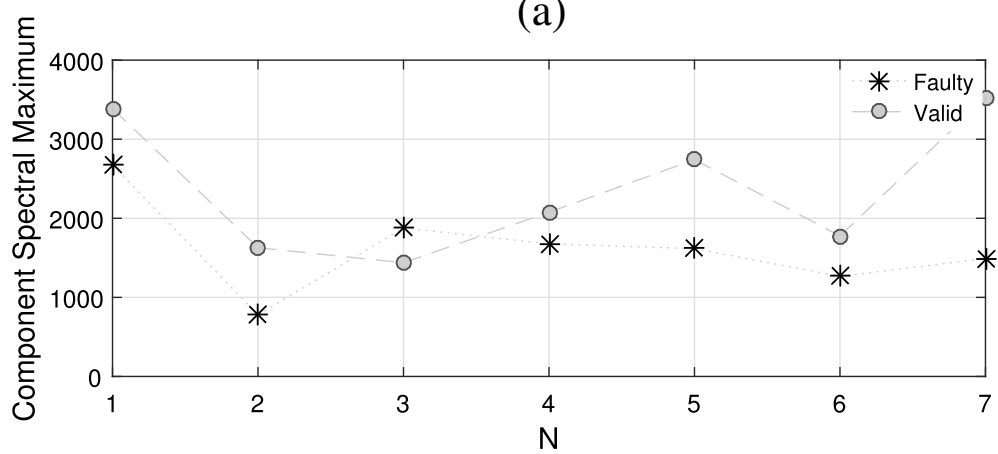

(b)

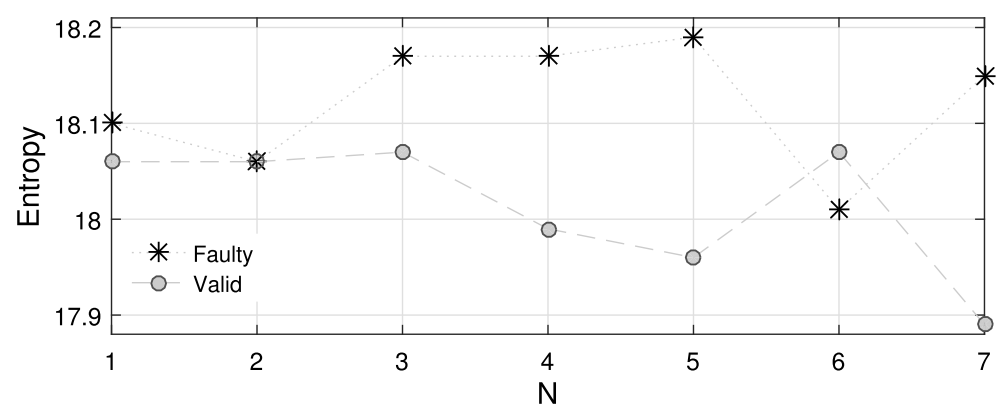

(c)

Fig. 3 a Signal energy, b spectrum maximum value inside components' bandwidth, and c global entropy spectrogram's entropy value for measured electrodes

in order to minimize the within-cluster sum of squares, with the minimal sum:

$$
\underset{C_{n}}{\operatorname{argmin}} \sum_{k=1}^{K} \sum_{\rho_{n}(p, m) \in C_{n, k}}\left\|\rho_{n}(p, m)-\mu_{n, k}\right\|^{2},
$$

where $\mu_{n, k}$ is the mean of each set $C_{n, k}$.

Thus, $K$ classes $\rho_{n, k}(p, m), k \in \mathbb{N}, 1 \leq k \leq K$, derived from the TFD $\rho_{n}(p, m)$, are obtained as

$$
\rho_{n, k}(p, m)=\left\{\begin{array}{cc}
\rho_{n}(p, m), & \text { if } \rho_{n}(p, m) \in C_{n, k} \\
0, & \text { elsewhere. }
\end{array}\right.
$$

Results presented in this paper are obtained by the pre-processing $\mathrm{K}$-means algorithm computed using the parameter $K=5$.
Classes containing mainly noise will present significantly larger time-frequency supports and smaller coefficients, when compared to classes containing useful information (since noise is flat in the TF plane while components are prominent ridges) [32]. Thus, it is reasonable to expect that data originated from the signal component will be predominant in the classes marked by higher values of the parameter $k$, while smaller values of $k$ will mark classes containing mainly noise-originated data. In the light of the above, the denoising procedure is performed by discarding all classes but the one marked by the largest value of the parameter $k(k=K)$. Data contained in $\rho_{h, K}[p, m]$ are assumed to be output of a strict denoising procedure, simplifying the assessment of the component's continuity. 

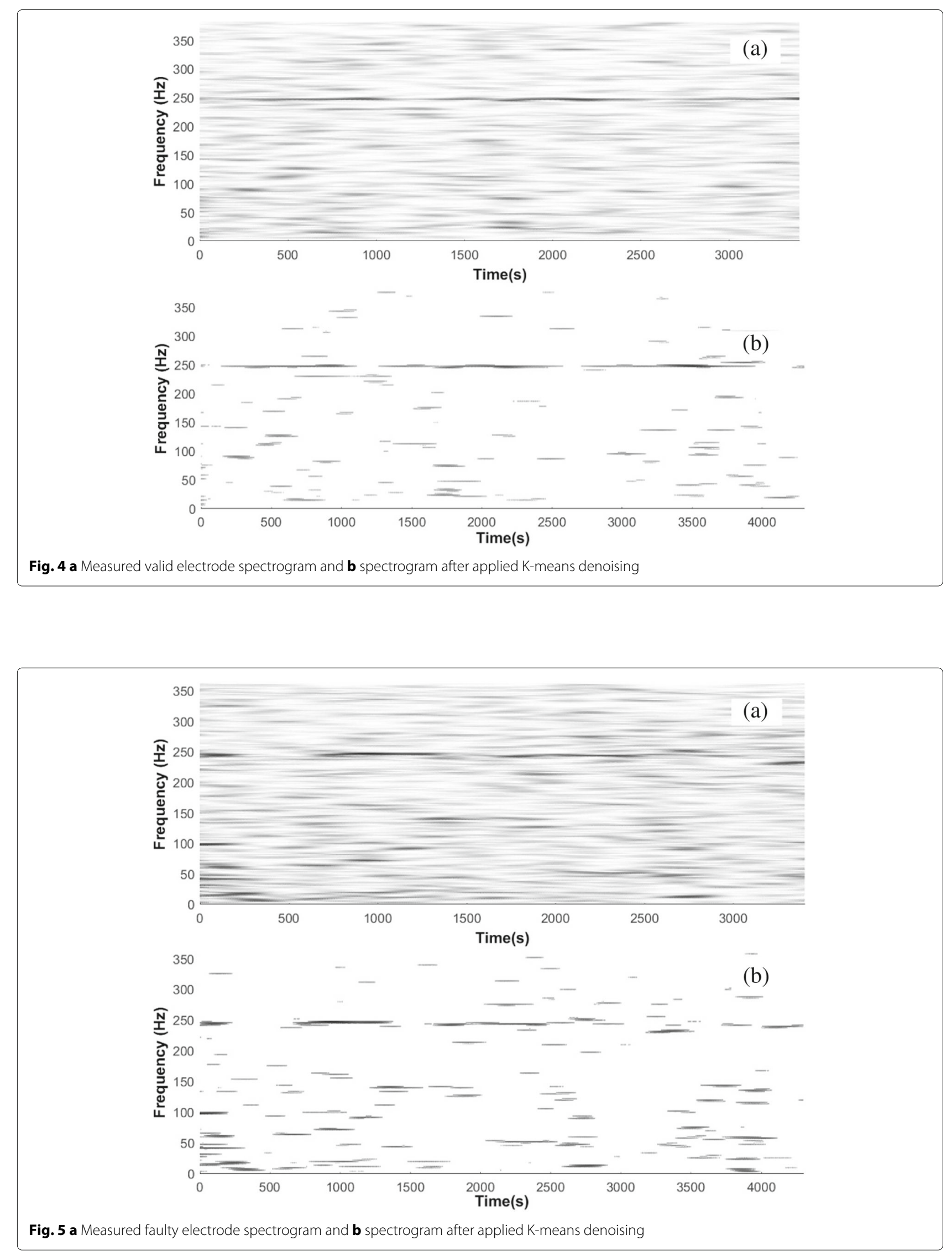


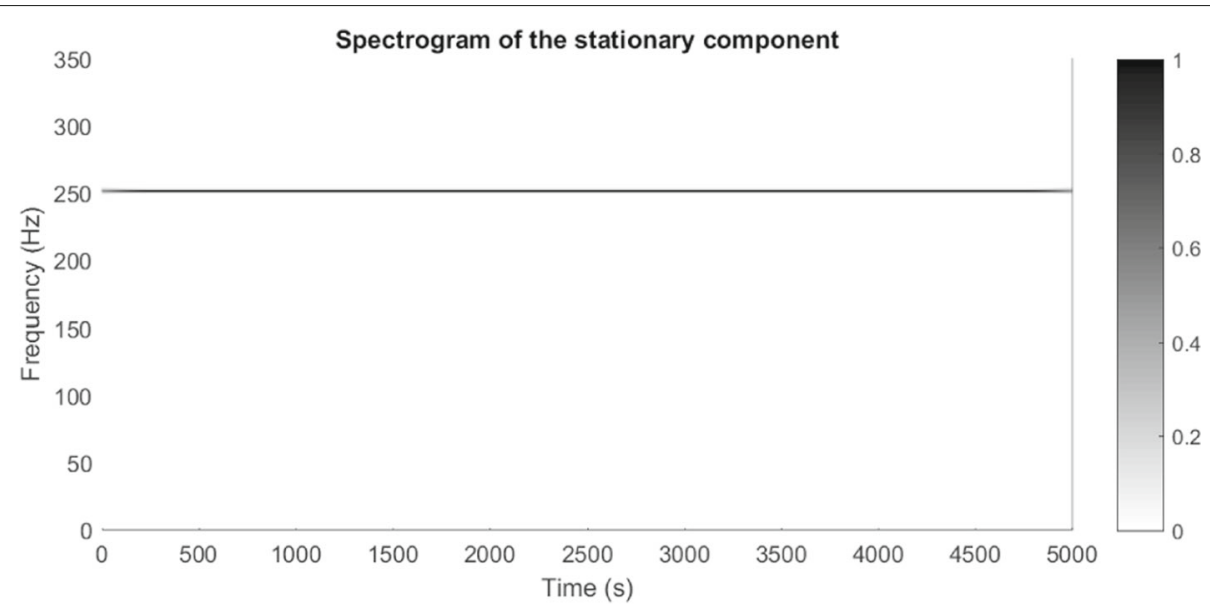

Fig. 6 Synthetic component added around $250 \mathrm{~Hz}$

Next, a narrow frequency bandwidth of the TF plane of $\rho_{n, K}[p, m]$, around the stationary components' frequency, is analyzed to determine the component's continuity by connected components labeling in a binary image (2D). Connected-component labeling or region extraction is an algorithmic application of graph theory, where subsets of a given data set are uniquely labeled based on predefined conditions [33]. The algorithm returns a matrix of the same size as the input matrix $\rho_{n, K}[p, m]$, containing labels for the connected components which are then used to extract relevant information from the input $\rho_{n, K}[p, m]$. Each individual separated set of data in the TFD is given a unique label, in a form of a matrix mask, which enables the component extraction procedure. The continuity is quantified through the size (length) of the clusters the stationary component is composed of, where the longest cluster is considered as a measure of the electrodes' validity. After a narrow frequency band around the stationary component has been designated, the cluster length $L_{\max }(n)$ is determined as the maximal number of consequent (neighboring) non-zero coefficients scanning in the time axis direction.

In Figs. $4 \mathrm{~b}$ and $5 \mathrm{~b}$ it can be seen that the component is preserved better in the case of higher SNR (valid electrode), presenting a continuous energy ridge in the TF plane, unlike in the case of lower SNR (faulty electrode),

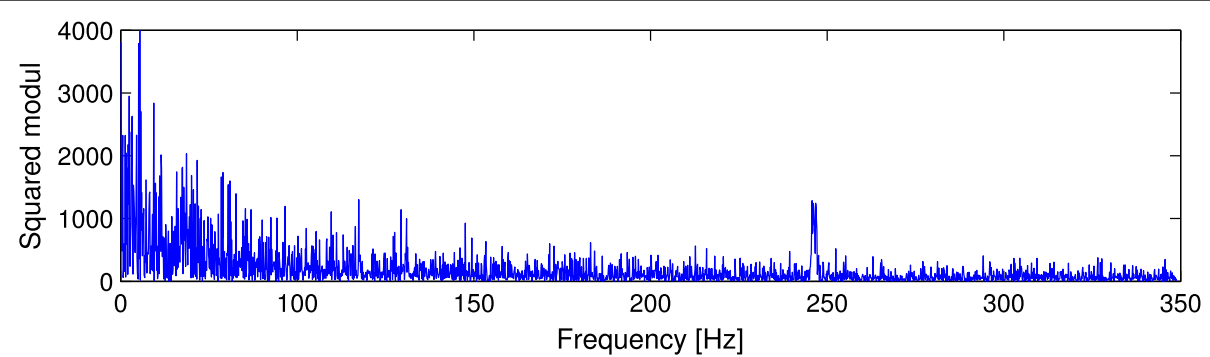

(a)

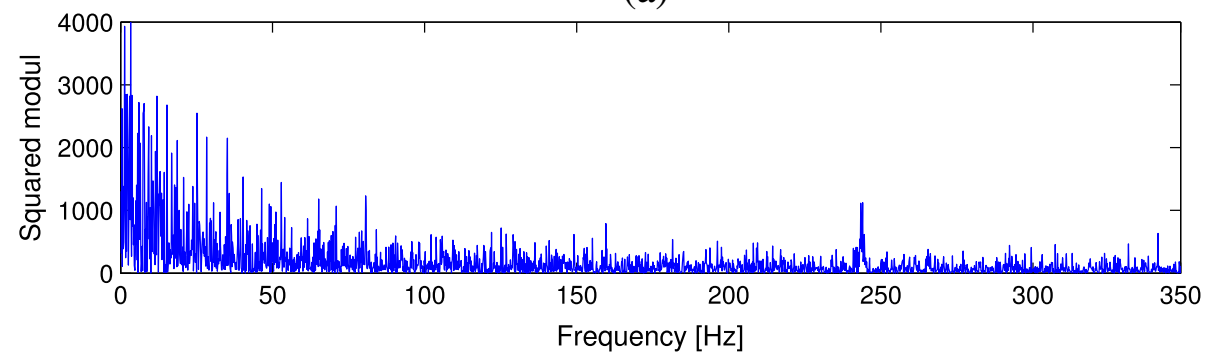

(b)

Fig. 7 Simulated signal energy spectral densities for $\mathbf{a}$ valid electrode and $\mathbf{b}$ faulty electrode 

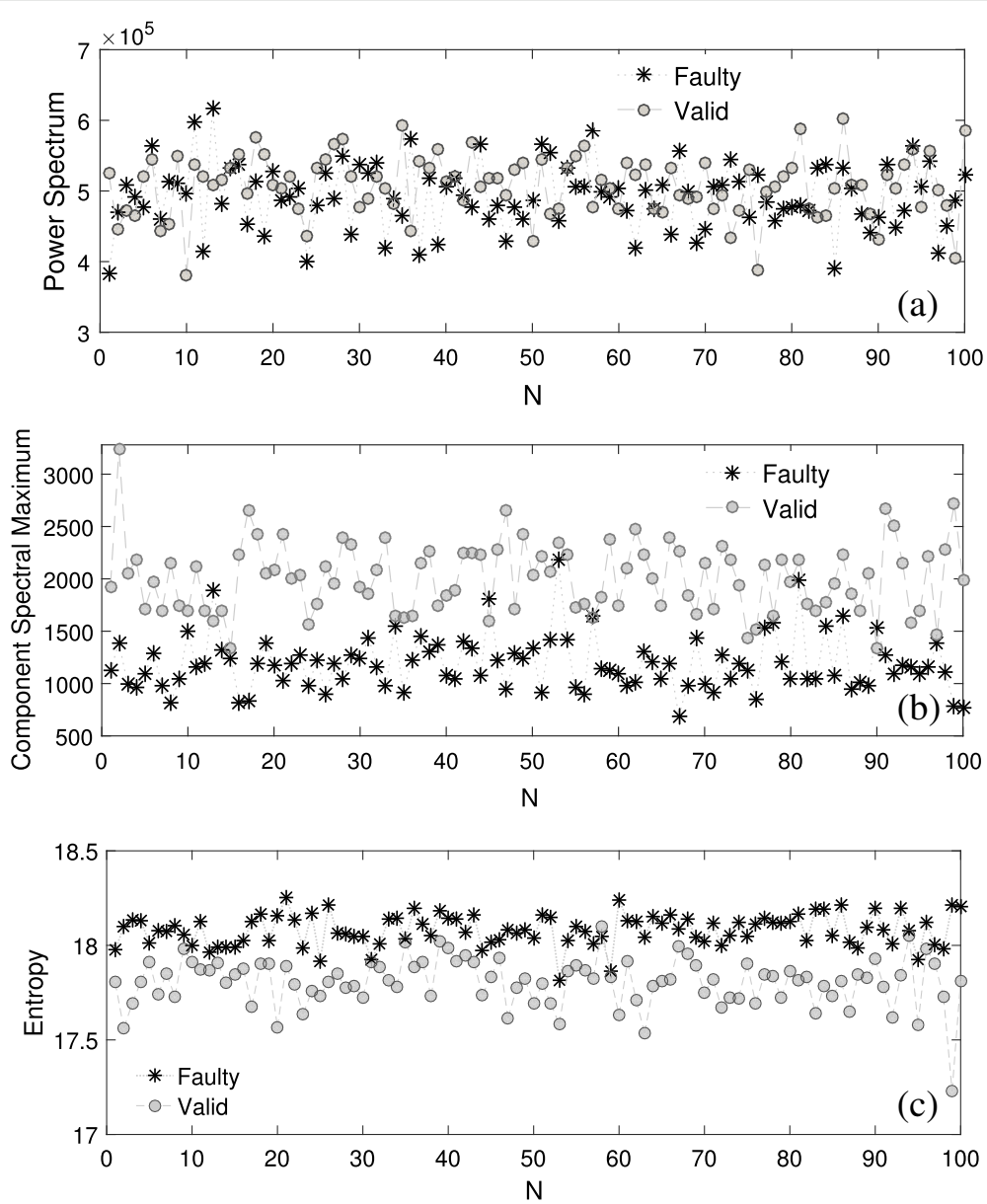

Fig. 8 Simulated results for a signal energy, b spectrum maximum value inside components' bandwidth, and c global entropy value for simulated electrodes

where the component energy appears broken and uneven inside its bandwidth.

\section{Criteria application on simulated data}

We have developed a model that fits distributions of signal energies, valid electrode energy spectral densities' local maxima, and Renyi entropy of the spectrogram of measured data. The developed computer model has been applied in [27]. It is known from the literature that the resistance fluctuations can be characterized as a superposition of the flicker and white noise $[3,6]$. Noise-to-noise and signal-to-noise ratios are determined using the average signal energy and average global entropy of the measured signal spectrograms. Flicker and white Gaussian noises are superimposed in noise-to-noise ratio of $7 \mathrm{~dB}$. The mixture of flicker and white noise is added to the stationary components with $\mathrm{SNR} \approx-16 \mathrm{~dB}$ for the valid electrode, and $\mathrm{SNR} \approx-18 \mathrm{~dB}$ for the faulty electrode. The stationary component, embedded in the previously described noise mixture, is centered around the fifth network harmonic at $f_{0}=250 \mathrm{~Hz}$, Fig. 6. It is defined as:

$$
y=\cos \left(2 \pi f_{0}(p-P / 2)\right) .
$$

Figure 7 shows the energy spectral densities of the resistance fluctuations of the simulated electrodes provided by the computer model, with the stationary component. It can be seen that no significant difference between the spectra of valid and faulty electrodes can be observed. Figure 8a reports the signal energies, calculated using the energy spectral densities, of the simulated valid and faulty electrodes, respectively, confirming that discerning of valid and faulty electrodes is not possible.

Figure $8 \mathrm{~b}$ shows the energy spectral densities' maxima, inside the stationary component's bandwidth, of simulated valid and faulty electrodes, and Fig. 8c shows the entropy values of the signals spectrograms. Figures 9 and 10 show the spectrograms of one simulated valid and one faulty electrode, before and after the denoising procedure, respectively. Figure 11 reports lengths of the longest 

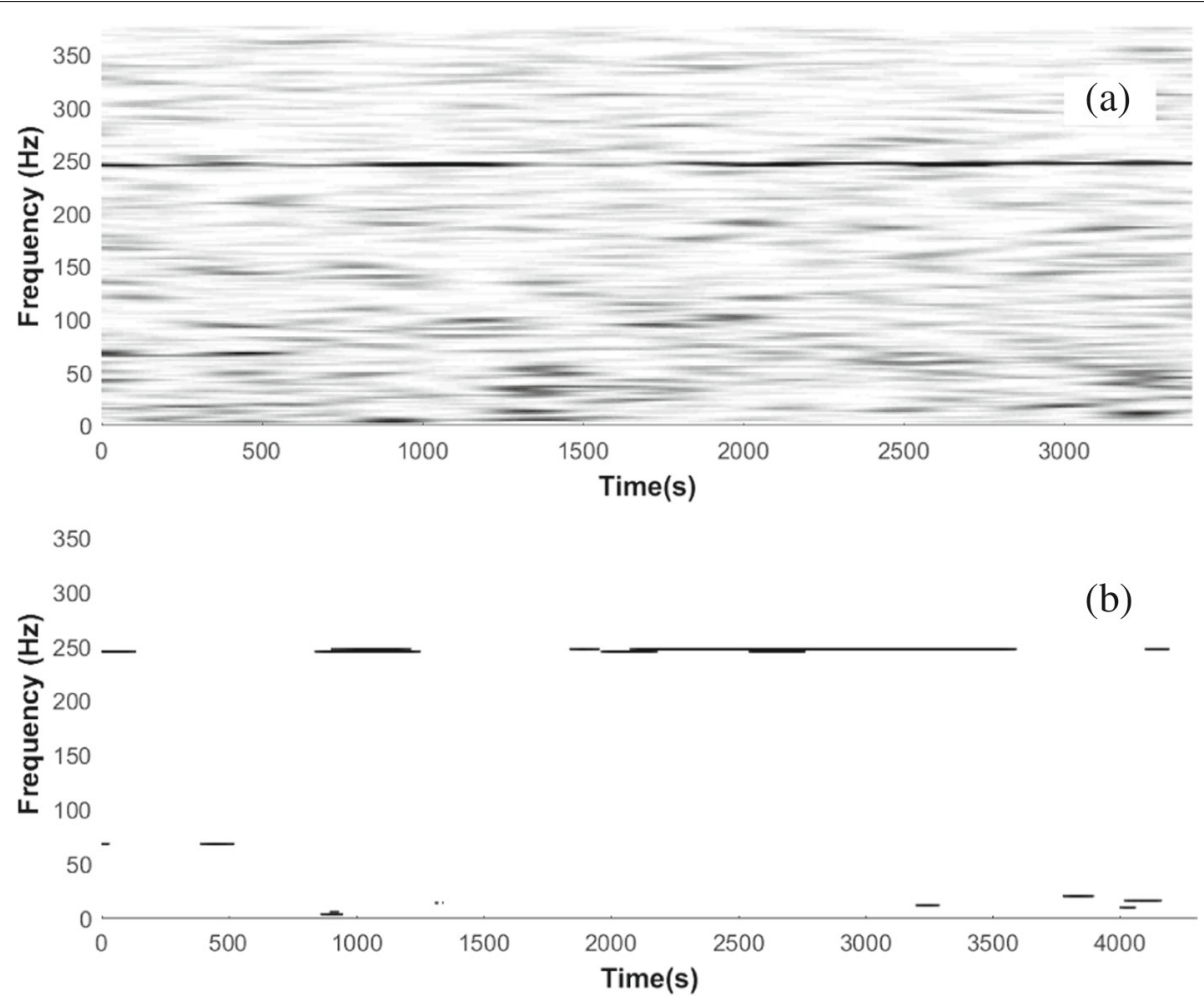

Fig. 9 a Simulated valid electrode spectrogram and $\mathbf{b}$ spectrogram after applied K-means denoising
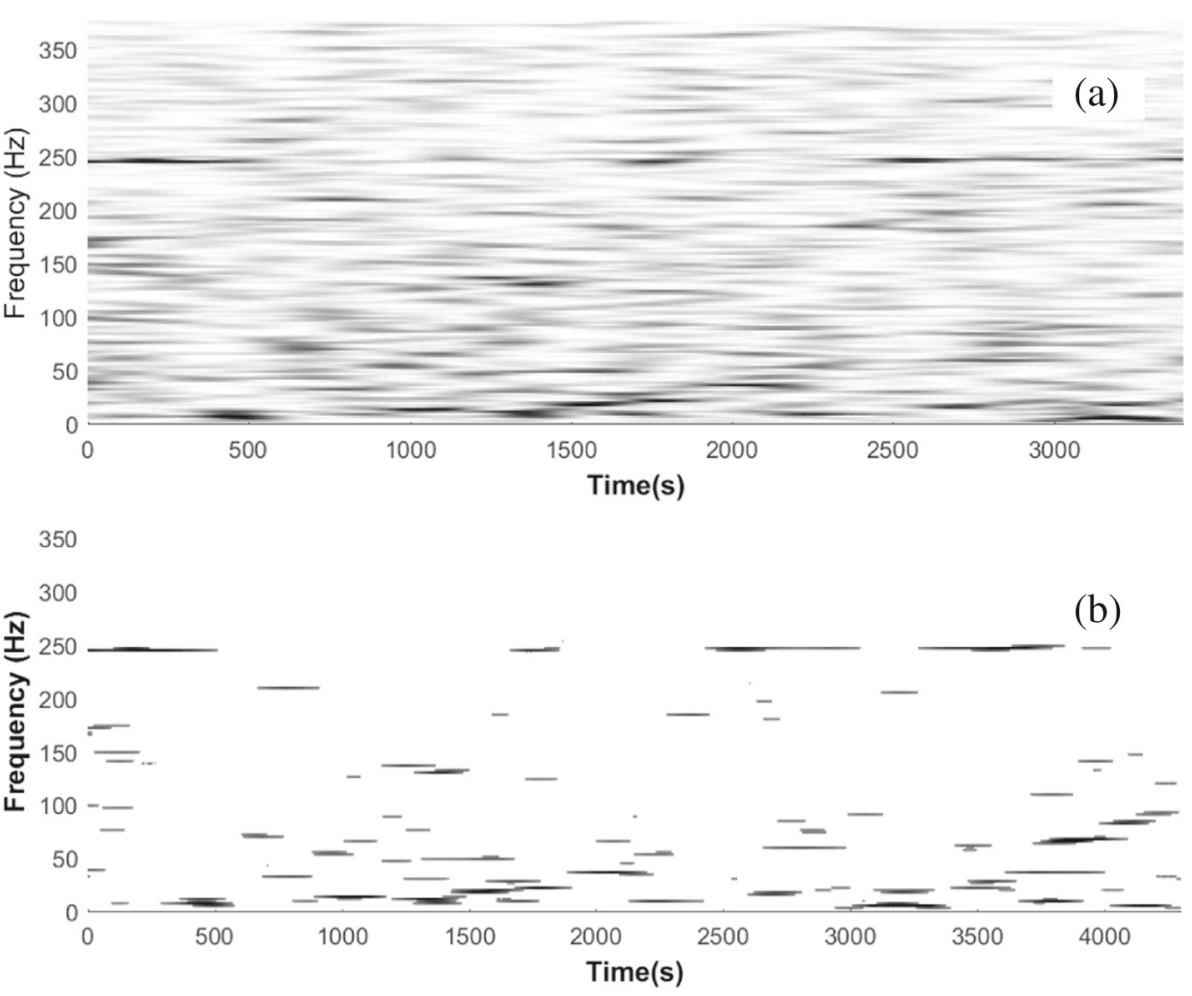

Fig. 10 a Simulated faulty electrode spectrogram and $\mathbf{b}$ spectrogram after applied K-means denoising 


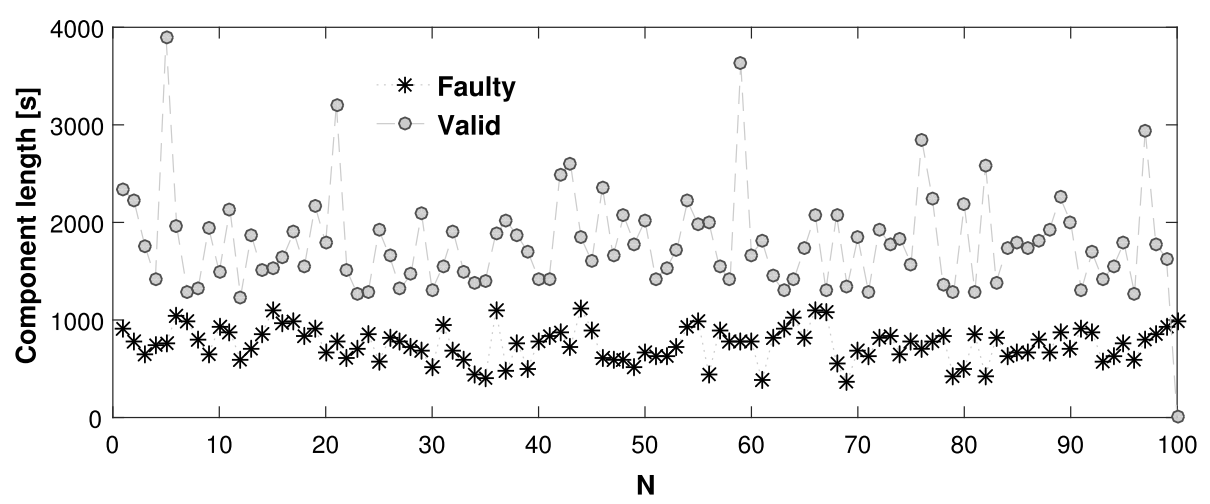

Fig. 11 The longest component length $L_{\max }(n)$ in the spectrogram of faulty and valid electrodes for simulated signals

energy clusters $L_{\max }(n)$, between the two groups of simulated signals, suggesting that precise classification could be obtained by the application of the proposed criterion. To validate the proposed classification criterion we have applied it to measured data, Fig. 12. In Fig. 12, no overlapping of the two data sets (valid and faulty) can be observed.

\section{Results and discussion}

Analysis of the spectral parameters of measured and simulated data shows the inadequacy of the classic spectral analysis in defining an unambiguous classification criterion of signals w.r.t. a small difference in SNRs. The signal energies of faulty and valid electrodes result in similarly distributed data (Fig. 3a, Fig. 8a), disqualifying energy detection [8-11] as potential classifier between valid and faulty electrodes.

As intuitively expected, in the case of higher SNR (valid electrodes), the component peaks are generally better preserved; however, opposite cases are not rare (Fig. 3b, Fig. 8b, Table 1.). The spectrogram shows a tendency of signals in higher SNR to present a more ordered structure, generally gaining smaller entropy values (Fig. 3c, Fig. 8c), result that indicates that smaller SNRs preserve a more compact structure of the component energy cluster. By exploiting the spectrogram ability of representing signal components as continuous energy concentrations, while noise effects can be described as sparse energy peaks in the TF plane, the time duration of the largest energy cluster has been found highly indicative of the SNR level. In

Table 1 Classification criteria performance evaluation (TP-true positives, TN-true negatives, FP-false positives, FN-false negatives)

\begin{tabular}{lllllllll}
\hline Measure & TP & TN & FP & FN & Acc. & Prec. & Recall & F1 \\
\hline Entropy & 107 & 15 & 92 & 0 & 0.570 & 0.537 & 1 & 0.698 \\
Spec. max. & 107 & 18 & 89 & 0 & 0.584 & 0.545 & 1 & 0.705 \\
Comp. len. & 107 & 102 & 5 & 0 & 0.976 & 0.955 & 1 & 0.977 \\
\hline
\end{tabular}

fact, lower SNRs result in prominent energy peaks with small time supports in the TF plane, as a result of the disordered spectrogram structure of noisy signals. On the other hand, in the case of higher SNRs, the component ridge consistently presents dominant TF peaks, since it is the TF dominant structure. As shown in Fig. 11 and 12, no overlapping in the lengths of the longest energy cluster $L_{\max }(n)$ between the two groups of signals has occurred, encouraging further testing of the proposed criterion.

In order to propose an improved criterion for discrimination of valid from faulty electrodes, the three measures that have shown potential employment as classification criteria, namely the energy spectral densities' maxima inside the stationary component's bandwidth, the spectrogram's entropy, and the length of the spectrogram's longest energy cluster, will be extensively tested on measured and simulated data.

In order to discern two groups of electrodes based on input data obtained for each of the three compared measures (Figs. 3b, and 8b, Figs. 3c, and 8c, Fig. 11, and 12), again the one-dimensional clustering $\mathrm{K}$-means method is applied as follows [30]: considering an $1 \times N$-dimensional set of observations

$$
C=\left\{L_{\max }(n) \mid n=1, \ldots, N\right\}
$$

the K-means algorithm partitions these $1 \times N$ observations into $K=2$ subsets

$$
C=\left\{C_{k} \mid k \in \mathbb{N}, 1 \leq k \leq K\right\},
$$

as

$$
\underset{C}{\operatorname{argmin}} \sum_{k=1}^{2} \sum_{L_{\max }(n) \in C_{k}}\left\|L_{\max }(n)-\mu_{k}\right\|^{2},
$$

where $\mu_{k}$ is the mean of each set $C_{k}$. In the presented case, the set $C$ has to be partitioned into two subsets, as:

$$
C=\left\{C_{1}, C_{2}\right\}
$$

with $C_{1}$ containing cluster lengths $L_{\max }(n)$ estimated to be responses of faulty electrodes, while $C_{2}$ containing cluster 


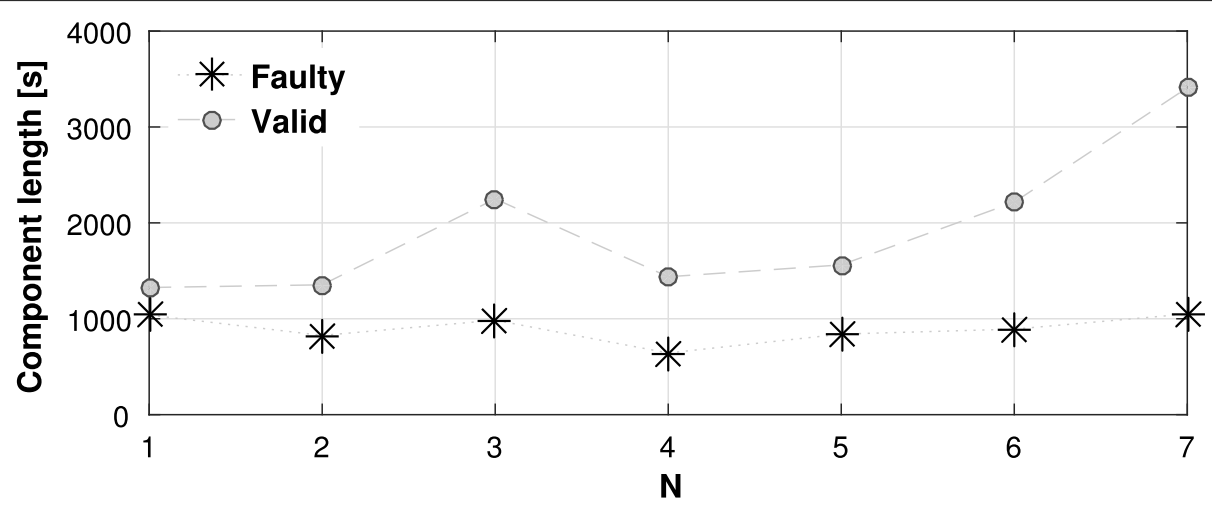

Fig. 12 The longest component length $L_{\max }(n)$ in the spectrogram of faulty and valid electrodes for measured signals

lengths $L_{\max }(n)$ estimated as responses on valid ones. Based on the elements' indexes $\mathrm{n}$ of the subsets $C_{1}$ and $C_{2}$, two sets are obtained, $S_{f}$ containing indexes $n$ of faulty electrodes, and $S_{v}$ containing indexes $n$ of valid electrodes. In Fig. 12, which reports the lengths of the longest component in the spectrogram of faulty and valid electrodes for measured signals, it can be seen that a substantial difference in of the proposed estimate can be found between the two groups of signals. Table 1 reports the performance evaluation of the three classification criteria in terms of accuracy, precision, recall and F1 score, where it can be observed that the classification criterion based on the maximal component length provides optimal fault detection procedure. A graphical user interface (GUI) has been developed in order to simplify and speed up the process of failure detection, Fig. 13. GUI output consists of data sufficient to discard a faulty electrode or accept the valid one, including a graphical representation of detected useful data in analyzed signals spectrogram. The proposed failure algorithm is described in a flowchart shown in Fig. 14. The spectrogram has been used for the signals' representations as one of the most commonly used TFDs in practical applications, since it is cross-terms free, characterized by realness, and strict positivity. However, other TFDs with enhanced representation performance, as the S-method [34] or Modified B Distribution [35], should be considered for future work. The K-means has been used as denoising method since it operates as $1 \mathrm{D}$ segmentation

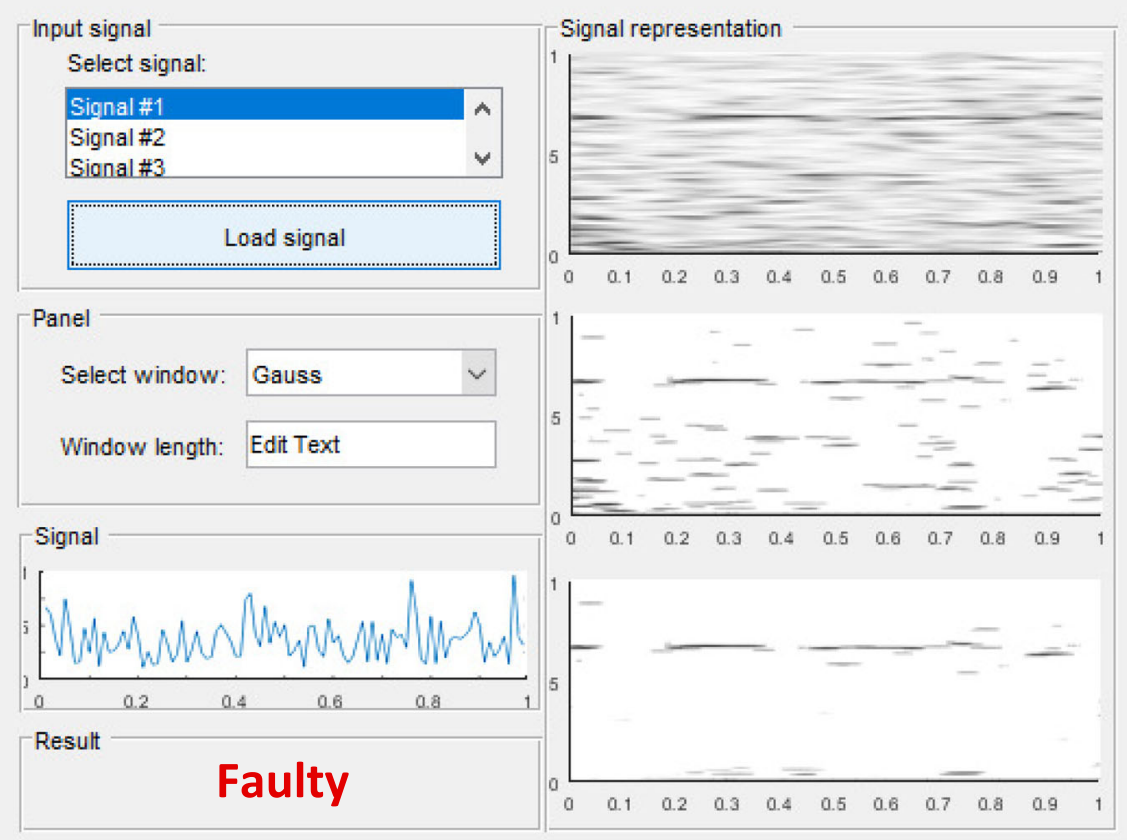

Fig. $13 \mathrm{GUI}$ of software tool prototype of a computer-aided decision system 


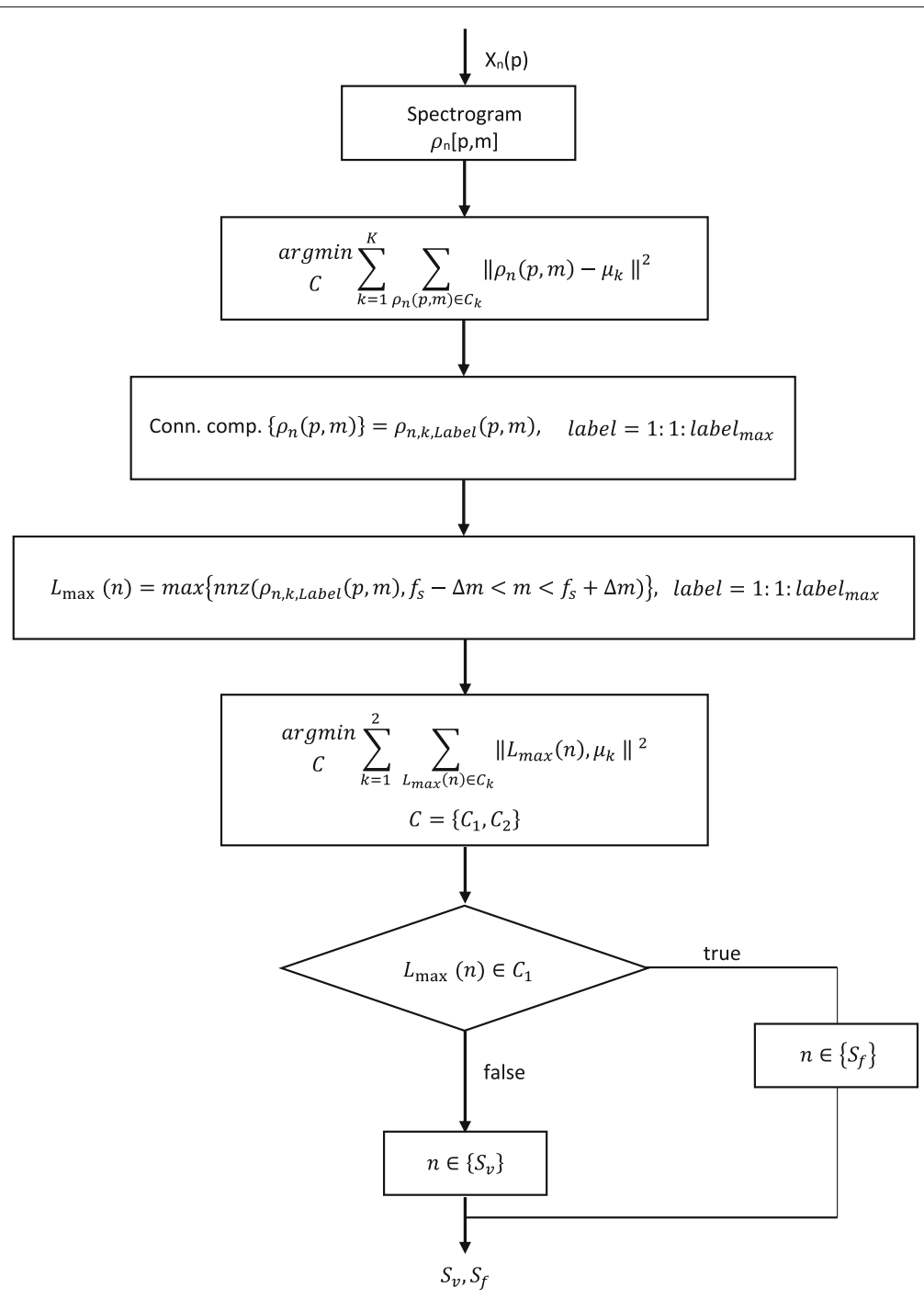

Fig. 14 Flowchart of the proposed algorithm

procedure directly on the TFD, taking into account the difference in the structural features of noise, being flat in the TFD, and signal components, being elongated energy regions. Future work will include optimization of the number of classes $\mathrm{K}$ utilized in the K-means algorithm, in order to emphasize the component's continuity in the denoised TFD .

\section{Conclusion}

In this paper, the possibility of distinguishing signals presenting slightly different power ratios of useful information content (i.e., signal component) and noise, using methods of spectral and time-frequency analysis is investigated. It has been observed that the analyzed signals, obtained from the responses of valid and faulty electronic devices (EEG electrodes), due to slightly different levels of noise (lower SNR in faulty and higher SNR in valid electrodes), present some minor differences in their spectral characteristics. The energy spectral density is not indicative of the SNR, and the spectral peak of the component tends to be preserved better in signals with higher SNR. However, this information appears quite unstable, leading to a significant overlapping of results obtained from faulty and valid electrodes, resulting in a significant of classification error. A new insight in the signal structure is provided by the time-frequency signal representation, by computing the signal spectrogram. The spectrogram entropy indicates a general better preservation of the component structure in higher SNRs, however, representing alone a weak classification criterion. The better preservation of the component in higher SNR is assessed by the length of the longest continuous energy cluster in the time-frequency domain. Using this criterion, a reliable classification method has been established providing 
an effective faulty electrode detection procedure, with an error rate of less than $3 \%$, which can potentially help in reducing the possibility of misreading EEG signals by medical personnel. The importance of developing the failure detection method can be deduced from the fact the research has been commissioned by the manufacturer due to the feed back from medical personnel, noticing the appearance of artifacts produced by cold solder.

\section{Abbreviations}

AWGN: Additive white Gaussian noise; DC: Direct current; DUT: Device under testing; EEG: Electroencephalogram; FN: Flicker noise; GUI: Graphical user interface; SMU: Source measure unit; SNR: Signal-to-noise ratio; TF: Time frequency; TFD: Time-frequency distribution

\section{Authors' contributions}

ZM developed the software solution with GUI for the proposed computer analysis and research based on the signal processing methods developed and designed by NS. IM designed the measurement setup and led measurement procedure for obtaining input signals for the developed sofware solution. DS advized the team on research methods and results' interpretation. All authors read and approved the final manuscript.

\section{Funding}

Ministry of Science and Education of the Republic of Croatia.

\section{Availability of data and materials}

Please contact the author for data requests.

\section{Ethics approval and consent to participate}

Not applicable.

\section{Consent for publication}

Not applicable.

\section{Competing interests}

The authors declare that they have no competing interests.

\section{Author details}

${ }^{1}$ Faculty of Engineering, "Juraj Dobrila" University of Pula, Pula, Croatia. ${ }^{2}$ University of Split Faculty of Electrical Engineering, Mechanical Engineering and Naval Architecture, University of Split, Split, Croatia. ${ }^{3}$ Faculty of Electrical Engineering and Computing, University of Zagreb, Zagreb, Croatia.

Received: 16 May 2019 Accepted: 11 July 2019

Published online: 27 August 2019

\section{References}

1. D. Kwon, M. H. Azarian, in Proceedings of 59th Electronic Components and Technology Conference. Detection of Solder Joint Failure Precursors on Tin-Lead and Lead-Free Assemblies using RF Impedance Analysis (IEEE, San Diego, 2009), pp. 663-667

2. J. Pan, J. Silk, in Proceedings of 44th International Symposium on Microelectronics. A Study of Solder Joint Failure Criteria, (Long Beach, California, 2014)

3. I. Marasović, Z. Milanović, I. Zulim, Modelling and detection of failure in medical electrodes. Automatika. 60(1), 1-9 (2014)

4. C. Pennetta, L. Reggiani, G.y. Trefan, F. Fantini, A. Scorzoni, I. De Munari, Investigation of the role of compositional effects on electromigration damage of metallic interconnects. Comp. Mater. Sci. 22, 13-18 (2001)

5. Z. Tang, F. G. Shi, Stohastic simulation of electromigration failure of flip chip solder bumps. Microele. J. 32, 53-60 (2001)

6. A. Bora, A. K. Raychaudhuri, Evolution of $1 / f^{\alpha}$ noise during electromigration stressing of metal film: Spectral signature of electromigration process. J. Appl. Phys. 66, 113701 (2006)

7. N. E. Hakiki, M. F. Montemor, M. G. S. Ferreira, M. da Cunha Belo, Semiconducting properties of thermally grown oxide films on AISI 304 stainless steel. Corros. Sci. 42, 687-702 (2000)
8. H. Urkovitz, Energy detection of unknown deterministic signal. Proc. IEEE. 55(4), 523-531 (1967)

9. S. Atapattu, C. Tellambura, H. Jiang, Energy detection for spectrum sensing in cognitive radio. (Springer, New York, 2014)

10. S. A. Chouakri, M. Slamat, A. Taleb-Ahmed, in Proceedings of the Fourth International Conference on Advanced Information Technologies and Applications (ICAITA 2015). Implementation of a BPSK Modulation Based Cognitive Radio System Using the Energy Detection Technique (AIRCC, Dubai, 2015)

11. S. P. Herath, N. Rajatheva, C. Tellambura, in Proceedimgs of Canadian Conference on Electrical and Computer Engineering. On the energy detection of unknown deterministic signal over Nakagami channels with selection combining, (2009)

12. G. Yu, S. Mallat, E. Bacry, Audio denoising by time-frequency block thresholding. IEEE Transactions on signal processing. 56(5), 18301839 (2008)

13. S. Stankovi, S. Krishnan, B. Mobasseri, Y. Zhang, Time-Frequency Analysis and Its Applications to Multimedia Signals. EURASIP J. Adv. Signal Process. 2010, 739017 (2010)

14. R. Steinberg, D. OShaughnessy, in Proceedings of IEEE International Conference on Acoustics, Speech and Signal Processing (ICASSP). Segmentation of a speech spectrogram using mathematical morphology (IEEE, Las Vegas, 2008)

15. H. Hassanpour, M. Mesbah, B. Boashash, Time-frequency feature extraction of newborn EEG seizure using SVD-based techniques. EURASIP J. Adv. Signal Process. 2004, 898124 (2004)

16. L. F. Chaparro, A. Akan, S. I. Shah, L. Durak-Ata, Applications of time-frequency signal processing in wireless communications and bioengineering. EURASIP J. Adv. Signal Process. 2010, 879104 (2010)

17. I. Orović, S. Stanković, T. Chau, C. M. Steele, E. Sejdić, Time-frequency analysis and Hermite projection method applied to swallowing accelerometry signals. EURASIP J. Adv. Signal Process. 2010, 323125 (2010)

18. F. Millioz, N. Martin, in Acoustics and Vibration, Thirteenth International Congress on Sound and Vibration, ICSV13, Vienna, Austria, 2-6 July. Time-frequency segmentation for engine speed monitoring, Special session on Pattern Recognition (Curran Associates, Inc., New York, 2006)

19. B. Boashash, Time frequency signal analysis and processing: a comprehensive reference. (Elsevier Academic Press, Cambridge, 2016)

20. F. Hlawatsch, G. F. Boudreaux-Bartels, Linear and quadratic time-frequency signal representation. IEEE Sig. Process. Mag. 9(2), 21-67 (1992)

21. S. Kadambe, G. F. Boudreaux-Bartels, A comparison of the existence of cross terms in the Wigner distribution and the squared magnitude of the wavelet transform and the short time Fourier transform. IEEE Trans. Sig. Process. 40(10), 2498-2517 (1992)

22. A. Rényi, in Proceedings of 4th Berkeley SympMathematics of Statistics and Probability. On measures of entropy and information (Univ. of Calif. Press, 1961), pp. 547-561

23. R. Baraniuk, P. Flandrin, A. Janssen, O. Michel, Measuring time-frequency information content using the Rényi entropies. IEEE Trans. Inf. Theory. 47(4), 1391-1409 (2001)

24. R. Baraniuk, P. Flandrin, O. Michel, in Proceedings of IEEE International Confonference on Acoustics, Speech, and Signal Processing-ICASSP94. Time-frequency complexity and information (IEEE, Adelaide, 1994), pp. 329-332

25. S. Aviyente, W. J. Williams, Minimum Entropy Time-Frequency Distributions. IEEE Signal Processing Letters. 12(1), 37-40 (2005)

26. V. Sucic, N. Saulig, B. Boashash, Estimating the number of components of a multicomponent nonstationary signal using the Short-term time-frequency Rényi entropy. EURASIP J. Adv. Signal Process. 2011, 125 (2011)

27. I. Marasović, N. Saulig, Ž. Milanović, in Proceedings of the 23rd International Conference on Software, Telecommunications and Computer Networks (SoftCOM 2015), Bol, Croatia, FESB. Rényi entropy based Failure detection of medical electrodes (University of Split, Croatia, 2015)

28. B. Boashash, Estimating and interpreting the instantaneous frequency of a signal-part 1Fundamentals. Proc. IEEE. 80(4), 520-538 (1992)

29. B. Boashash, Interpreting and estimating the instantaneous frequency of a signal-part 2: algorithms and applications. Proc IEEE. 80(4), 540-568 (1992)

30. I. E. Frank, R. Todeschini, The data analysis handbook. (Elsevier, New York, 1994) 
31. Ž. Milanović, N. Saulig, Nicoletta, V. Sucic, in Proceedings of International Conference on Innovative Technologies (IN-TECH 2013). Nonstationary signal blind source separation using clustering algorithms, (Budapest, 2013)

32. N. Saulig, Ž. Milanović, C. loana, A local entropy-based algorithm for information content extraction from time-frequency distributions of noisy signals. Digit. Sig. Process. 70, 155-165 (2017)

33. M. B. Dillencourt, H. Samet, M. Tamminen, A general approach to connected-component labeling for arbitrary image representations. J. ACM (JACM). 39(2), 253-280 (1992)

34. L.J. Stanković, A method for time-frequency signal analyisis. IEEE Trans. Sig. Process. 42(1), 225-229 (1994)

35. Z. M. Hussain, B. Boashash, Adaptive instantaneous frequency estimation of multicomponent FM signals using quadratic time-frequency distributions. IEEE Trans. Sig. Process. 50(8), 1866-1876 (2002)

\section{Publisher's Note}

Springer Nature remains neutral with regard to jurisdictional claims in published maps and institutional affiliations.

\section{Submit your manuscript to a SpringerOpen ${ }^{\circ}$ journal and benefit from:}

- Convenient online submission

- Rigorous peer review

- Open access: articles freely available online

- High visibility within the field

- Retaining the copyright to your article

Submit your next manuscript at $>$ springeropen.com 